\title{
Cronología de la erupción dentaria permanente en pacientes con síndrome de Down
}

\author{
Lilian Jara S. ${ }^{1}$; Alejandro Ondarza G. ${ }^{2}$; \\ Rafael Blanco C.'
}

\section{Chronology of the eruption of the permanent dentition in patients with Down's syndrome}

\begin{abstract}
Two hi, ndred and forly patests with Cown symdrome tron Santiago, Chile, were examined to determine the eruption time and sequence of their permanent dentitior ir comparison with the oattern shown by normal chilean population. Boys with Down syndrome show sign, icicant delay in both maxillary and mandibular teeth eruption time. A.mong maxiliary teeth, eruotion was shown to be delayed in first right molar to $85.35 \pm 20.03$ months, in first eft rolar to $87.41=22.37$. first and second left premolars to $161.60 \pm 60.43$ and $172.10 \pm 79.57$ months respextively and left canire to $163.72 \pm 81.55$ mont:7s. In the mandible first right molar erupted at $90.98 \pm 24.52$ months, right and left central incisors at $84.26 \pm 21.38$ ard $84.59 \pm 17.72$ months respectivaly, right and left lateral incisors at $101.89 \pm 23.79$ and $117.53 \pm 83.02$ months respectively and the left canine at $147.57 \pm 41.54$ months. Girls with Down syndrome also had significant delays in the time of eruption which wias, for the maxillary

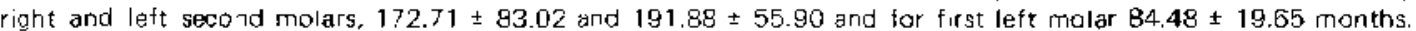
In the mancible, right second molar and first premolar eruption time delavs were docurrented to $163.30 \pm 59.31$ and $131.07 \pm 21.94$ morths anc aiso for risht and left atera! incisors to $102.27 \pm 52.86$ and $712.87 \pm 73.46$ months respectively. The sequence of upoer and tower dental eruption of bovs with Lown syndrome shows great asyncrony which affects twenty teeth $(71.4 \%)$ as combared with the normal population. In girls with Down svndrome this asvnchroric sequence al fects only 15 teeth (E3.6\%). One of the main differences between Down syrdrome and normal individ $\lrcorner$ als is due to the greater variance observed in the Down sample.

(Kev words: Down svidrome. teeth eruption, permanent dentition.)
\end{abstract}

Los tiempos de la erupción dentaria en el hombre varían normalmente tanto en la dentición temporal como en la definitiva. A pesar de ello la secuencia de erupción es similar en todas las razas. En el control de la erupción estarían implicados factores genéticos, ambientales y sistémicos, que tendrian relación con la presencia o ausencia de morfógenos que intervienen en los procesos de inducción primaria y secundaria de la odontogénesis y, por lo tanto, en la erup. ción dentaria ${ }^{1}$. El retraso de esta última se ha asociado con desórdenes sistémicos y se ha observado en los síndromes de Down y de Turner,

1. Departamento de Biología Celular y Genética, l-acultad de Medicina, División Ciencias Médicas Norte, Universidad de Chile.

2. Departamento de Morfología Experimental, Facuttad de Medicina, División Ciencias Médicas Norte, lonversidad de Chile. displasia cleidocraneal, atrofia hemifacial $y$ mucopolisacaridosis, entre otros ${ }^{2}$. En el sindrome de Down se ha observado retardo de la erupción en ambas detiticiones, comparado con la población normal ${ }^{3-17}$. En la dentición temporal se ha descrito retardo de la erupción de los incisivos y caninos en ambos maxilares y la serie temporal se completa alrededor de los cuatro o cinco años o después. Los tiempos de erupción de la dentición permanente son también irregulares. La extensión del retardo, comparado con nifios normales, no se ha estudiado ampliamen$\mathrm{t}^{6,8}$. Algunas publicaciones han analizado las diferencias en la erupción dentaria permanente al comparar niños Down con sus hermanos normales $^{13}$.

E1 objetivo del presente estudio fue determinar el patrón de erupción de la dentición per. manente en una muestra de niños chilenos con sindrome de Down y compararlo con el patrón de erupción de la población normal chilena. 


\section{Material y Métodos}

Los pacientes consindrome de Down fueron seleccionados al azar entre la población escolar asistente a las Escuelas Especiales E-497, F-86 y Coocendes de Santiago, Chile. La muestra estuvo formada por 240 pacientes, 124 niñas, cuyo matgen de edad era de 48 a 444 meses. La fecha de nacimiento de cada individuo se obruvo de los registros de la escuela respectiva. Las pacjentes se dividieron en 16 grupos de alad con intervalos de 12 meses entre ellos. El primer grupo correspondía a los 48 meses de eda đ̃ y el último incluyó a los pacientes con edades superiores a 229 meses. Esta clasificación permitió comparas la cronología obtenida con la descrita para población chilena nomal'

El examen clínico de la boca lo realizó un solo investigador (A.O.), con un procedimiento uniforme para todos los individuos, bajo condiciones adecuadas de iluminación, consignando la presencia o ausencia de piezas dentarias. Se consideró erupción de un diente cuando cualquier porción de la corona aparecía en la cavidad bucal. Los terceros molares fueron excluidos del análisis.

Se tabuló el universo por sexo y por grupo etario. Posteriormente se tabuló el número de dientes erupcionados en cada grupo para cada pieza dentaria. La proporción de dientes crupcionados en cada grupo etario se obtuvo de estos datos y se transformó on valores de probito usando un programa computacional. El análisis de probito es un método iterativo de aproximaciones sucesivas que proporciona el mejor ajuste lineal por el procedimiento de los mínimos cuadrados. El programa computacional también entregó el promedio de la edad de erupción, la desviación típica y el error típico ${ }^{10}$.

El tiempo de erupción de cada diente se comparó con un estudio para población normal realizado con el mismo procedimiento ${ }^{17}$. Se aplicó la prueba de Student con la corrección de Welch para calcular diferencias significativas entre los valores de edad de erupción entre niños con síndrome de Down y normales

\section{Resultados}

La tabla 1 resume la distribución por edad y sexo de la muestra de individuos con síndrome de Down. La tabla 2 presenta el promedio de edad de la erupción (en meses) de cada diente, con su desviación típica y error típico para n1ños y niñas. Los promedios cuyos errores típicos fueron muy grandes no se consideraron en los análisis posteriores.

En las nifas con sindrome de Down el proceso de erupción de la dentición permanente se inició con la siguiente secuencia cronológica: el primer diente en aparecer fue el primer molar superior derecho a los 70,57 meses, luego emer. gió el incisivo central derecho inferior a los 77,57
Tabla 1

Distribución por edad y sexo en una muestra de población escolar chjlena son sindrome de Down

\begin{tabular}{lrrr}
\hline $\begin{array}{l}\text { Edad } \\
\text { (meses) }\end{array}$ & Nirios & Niñas & $\begin{array}{r}\text { Ambos } \\
\text { sexos }\end{array}$ \\
\hline $48-60$ & 10 & 14 & 24 \\
$61-72$ & 8 & 8 & 16 \\
$73-84$ & 8 & 11 & 19 \\
$85-96$ & 5 & 8 & 13 \\
$97-108$ & 12 & 7 & 19 \\
$109-120$ & 7 & 8 & 15 \\
$121-132$ & 6 & 4 & 10 \\
$133-144$ & 1 & 12 & 13 \\
$145-156$ & 5 & 8 & 13 \\
$157-168$ & 1 & 3 & 4 \\
$169-180$ & 5 & 5 & 10 \\
$181-192$ & 9 & 1 & 10 \\
$193-204$ & 3 & 5 & 12 \\
$205-216$ & 8 & 4 & 11 \\
$217-228$ & 6 & 5 & 43 \\
$229-444$ & 22 & 21 & \\
& & & 240 \\
Total & 116 & 124 & \\
\hline
\end{tabular}

meses, la pieza siguiente fue el primer molar inferior izquierdo, a los 78,42 meses, y en cuarto lugar el primer molar superior izquierdo a los 84,48 meses. En los varones con sindrome de Down el proceso comenzó a los 85,35 meses сол el primer molar superior izquierdo, segujdo por el primer molar inferior a los 86,15 meses, luego el primer molar superior derecho a los $87,4 \mathrm{I}$ meses y en cuarto lugar el primer molar inferior derecho, a los 90,98 meses. Por lo tanto, la secuencia de la erupción dentaria permanente es diferente en niños y nif́as con sindrome de Down $\mathrm{y}$, además, se observan diferencias por sexo en las piezas del maxilar superior e inferior.

En la tabla 3 se presenta la secuencia de erupción en niños y nif́as con sindrome de Down y en la tabla 4 la de población chilena normal ${ }^{16}$. En la figura se comparan las secuencias de erupción dentaria en individuos con síndrome de Down y normales, indicándose, en los primeros, las piezas que aparecen con retraso estadísticamente significativo con respecto a los normales. Los nifios con S. de Down presentan, en comparación con las niñas, mayor número de dientes con retardo significativo en la aparición. 
Tabla 2

Promedios, desviaciones típicas y errores típicos en meses para la erupción permanente en una muestra de niños chilenos con síndrome de Down

\begin{tabular}{|c|c|c|c|c|c|c|c|c|c|}
\hline H.S.O. & Seko & $x$ & D. T. & E. T. & H.S.I. & Sexo & $x$ & D.T. & E.T. \\
\hline \multirow{2}{*}{$2^{\text {" molar }}$} & $v$ & 130.06 & 150,39 & 47,0005 & \multirow{2}{*}{$2^{5}$ molar } & $v$ & 151,81 & 29,21 & 19,1350 \\
\hline & $\mathbf{M}$ & $172.7 !$ & 83.02 & 16.9570 & & $\mathrm{M}$ & 195.88 & 55,90 & 17,2500 \\
\hline \multirow{2}{*}{ fes molitr } & $v$ & 85.35 & 20.03 & 4,6392 & \multirow{2}{*}{ yer motas } & $v$ & 87.41 & 22,37 & 4.8121 \\
\hline & M & 70.57 & 61,90 & 20,8826 & & $\mathrm{M}$ & 84.48 & 19,65 & 5,2159 \\
\hline \multirow{2}{*}{$2^{\circ}$ premsuolas } & $v$ & 122.45 & 152,23 & 40,9679 & \multirow{2}{*}{$2^{\circ}$ premolar } & $\mathbf{v}$ & 172.10 & 79,57 & 17.4613 \\
\hline & M & 141.60 & 87,27 & 19,6262 & & $\mathbf{M}$ & 146,56 & 76,36 & 17,0344 \\
\hline \multirow{2}{*}{ jel premolar } & v & 126,29 & 63,49 & 20,0219 & \multirow{2}{*}{ jer premolar } & $\mathrm{v}$ & 161,60 & 60,43 & 13.7126 \\
\hline & $M$ & 130,89 & 50.42 & 10.4336 & & M & 124,01 & 37,43 & 9,6576 \\
\hline \multirow{2}{*}{ Canino } & v & 145,02 & 91,42 & $18,505 ?$ & \multirow{2}{*}{ Caning } & $v$ & 163.72 & 81.55 & 15,4571 \\
\hline & $M$ & $137.4 ?$ & 86.75 & 17.3025 & & M & 99.43 & 105,66 & 38.3478 \\
\hline \multirow{2}{*}{ |m: dateral } & V & 128,61 & 113,22 & 21,9723 & \multirow{2}{*}{ Inc. lateral } & $v$ & 99,81 & 144,27 & 34,8949 \\
\hline & $\mathrm{y}$ & 123,63 & 70,50 & 13,5898 & & $\mathbf{M}$ & 109.69 & 80,96 & 16,0589 \\
\hline \multirow[b]{2}{*}{ Inc. esntra! } & $v$ & 132.55 & 99,03 & 28,7655 & \multirow{2}{*}{ Inc. central } & $v$ & 128,19 & 92,49 & $29, ? 022$ \\
\hline & $M$ & 89,04 & 94,23 & 22,0493 & & $M$ & 91,64 & 94,63 & 22.7715 \\
\hline \multirow{3}{*}{$\begin{array}{l}\text { Intisyus } \\
\text { Leiltrisl }\end{array}$} & $v$ & 84.26 & 21,38 & 4,8026 & \multirow{3}{*}{$\begin{array}{l}\text { lncisivo } \\
\text { central }\end{array}$} & $\mathrm{v}$ & 84,69 & 15,72 & 3.7764 \\
\hline & & & & & & & & & \\
\hline & M & $? 7.57$ & 142,36 & 36,9668 & & M & 50,80 & 134,51 & $40,9780^{\circ}$ \\
\hline \multirow{3}{*}{$\begin{array}{l}\text { Inctivise } \\
\text { taleras }\end{array}$} & $\mathrm{v}$ & 101.89 & 23,79 & 5,4336 & \multirow{3}{*}{$\begin{array}{l}\text { Incisise } \\
\text { lateral }\end{array}$} & $v$ & 117,53 & 83,02 & 15,1394 \\
\hline & & & & & & & & & \\
\hline & $M$ & 102.27 & 52.86 & 9,6953 & & $\mathbf{M}$ & 112.87 & 73.46 & 14,1643 \\
\hline \multirow[b]{2}{*}{ Cuninu } & $v$ & 140,45 & 102.16 & 23.1175 & \multirow[b]{2}{*}{ Canino } & $v$ & 147.57 & 41,54 & 9,6888 \\
\hline & $M$ & $\$ 3,99$ & {$[44,3]$} & $82.8341=$ & & $M$ & 77.68 & 118.95 & $58.2745^{*}$ \\
\hline \multirow{2}{*}{ ter presnolar } & v & 137,07 & 56.74 & 14,7502 & \multirow{2}{*}{ jer premolar } & $\mathrm{v}$ & 157.87 & 93,25 & 22,9388 \\
\hline & $\mathrm{H}$ & 131,03 & 21,94 & 6.1782 & & $M$ & 130,86 & 75,29 & 18,9265 \\
\hline \multirow{2}{*}{ 2" premolar } & $v$ & 147,98 & $160,5 \mathrm{~L}$ & 35.0010 & \multirow{2}{*}{$2^{\circ}$ premolar } & $v$ & 176,01 & 92.45 & 20.2255 \\
\hline & $M$ & 120,73 & 99,49 & 31,7832 & & $M$ & $31,4 ?$ & $* *$ & $* *$ \\
\hline \multirow{2}{*}{ Jer molas } & $v$ & 90.98 & 24.52 & 6.1317 & \multirow{2}{*}{ 1er molar } & $\mathrm{V}$ & 86,15 & 15.57 & $3,732 ?$ \\
\hline & $M$ & 42.53 & 147,34 & $49,1805^{\circ}$ & & $M$ & 78,42 & 20.11 & 6,6615 \\
\hline \multirow{2}{*}{20 uniglar } & $v$ & 128,76 & 114.94 & 33,0023 & \multirow{2}{*}{$2^{\circ}$ molar } & $v$ & 152,84 & 79,68 & 18.9370 \\
\hline & $M$ & 163,30 & $\$ 9,31$ & 14,0991 & & M & 156,54 & 82,61 & 18,7340 \\
\hline
\end{tabular}

* No considerado para análisis posteriores debido a su gran error típico.

* * Datos no ajustados a la Distribución Gaussiana.
H.S.D. = Hemiarcada superior derecha
D.T. = Desviación típica
H.S.L = Hemiarcada superior izquierda.
E.T. = Error típico.
H.1.D. = Hemiarcada inferior derecha.
$\mathrm{X}=$ Promedio
H.l.I. = Hemiarcada inferior izquierda.
$\mathrm{V}=$ Varones
$\mathrm{M}=$ Mujeres 
Tabla 3

Secuencia cronológica de erupción de la dentición permanente en una muestra de niños chilenos con síndrome de Down*

\begin{tabular}{|c|c|c|c|c|c|c|c|c|c|c|c|}
\hline \multicolumn{6}{|c|}{ Niños } & \multicolumn{6}{|c|}{ Niñas } \\
\hline H.S.D. & $x$ & D.T. & H.S.I. & $\mathbf{X}$ & D.T. & H.S.D. & $\mathrm{X}$ & D.T. & H.S.I. & $x$ & D.T. \\
\hline Ml & 85,35 & 20,03 & M1 & 87,41 & 22,37 & Ml & 70,57 & 61,90 & M1 & 84,48 & 19,65 \\
\hline PM2 & 122,45 & 152,23 & IL & 99,81 & 144,27 & IC & 89.04 & 94,23 & IC & 91,64 & 94.63 \\
\hline PM1 & 126,29 & 63,49 & IC & 128,19 & 92,49 & IL & 123,63 & 70,50 & $\mathrm{C}$ & 99,43 & 105,66 \\
\hline $\mathrm{IL}$ & $12 B, 61$ & 113,22 & M2 & 151,81 & 79,21 & PMI & 130,89 & 50,42 & $\mathrm{IL}$ & 109,69 & 80,96 \\
\hline $\mathbf{M} 2$ & 130,06 & 150,39 & PM1 & 161,60 & 60,43 & $C$ & 137,47 & 86,75 & PM1 & 124,01 & 37,43 \\
\hline IC & 132,55 & 99,03 & $c$ & 163,72 & 81,55 & PM2 & 141,60 & 87,27 & PM2 & 146,56 & 76,36 \\
\hline C. & 145,02 & 91,42 & PM2 & 172.10 & 79.57 & M2 & 172.71 & 83,02 & M2 & 195,88 & 55,90 \\
\hline H.I.D. & $\mathrm{x}$ & p.T. & H.I.I. & $x$ & D.T. & H.J.D. & $x$ & D.T & H.I.I. & $\mathrm{x}$ & D. T. \\
\hline IC & 84,26 & 21,38 & IC & 84,6 & 15,72 & M1 & 42,53 & 147,34 & PY2 & 31,47 & 451,72 \\
\hline M1 & 90,98 & 24,52 & M1 & 86,15 & 15.57 & $\mathrm{C}$ & 53,99 & 144,31 & IC & 50,80 & 134,51 \\
\hline IL & 101,89 & 23,79 & $\mathrm{IL}$ & 117,53 & 83,02 & IC & 77,57 & 142,36 & C & 77,68 & 118,95 \\
\hline M2 & 128,76 & 114,94 & $\mathrm{C}$ & 147,57 & 41,54 & IL & 102,27 & 52,86 & M1 & 78,42 & 20,11 \\
\hline PM1 & 137,30 & 56.74 & M2 & 152,84 & 79,68 & PM2 & 120.73 & 99,49 & Il & 112,87 & 73,46 \\
\hline c. & 140,45 & 102,16 & PMI & 157,87 & 93,25 & PM1 & 131,07 & 21,94 & PM1 & 130,86 & 75,29 \\
\hline PM2 & 147,98 & 160.51 & PM2 & 176,01 & 92,45 & M2 & 163,30 & 59,31 & M2 & 156,54 & 82.61 \\
\hline
\end{tabular}

* Edad en meses.

IC $=$ Incisivo central
IL $=$ Incisivo IateraI
C $=$ Canino
PM1 $=1^{\text {er premolar }}$
PM2 $=2^{\circ}$ premolar
M1 $=1^{\text {er molar }}$
M2 $=2^{\circ}$ molar

\section{Comentario}

Estos resultados indican que hay un retardo estadísticamente significativo en la aparición de algunos dientes en niños con $\mathrm{S}$. de Down. Sin embargo, la secuencia de la erupción en ellos no es completamente diferente de la población normal. Los dientes con menores diferencias en sus tiempos de erupción respecto a los normales fueron los primeros molares superiores e inferiores $y$ los incisivos centrales y laterales. Se podria postular que las alteraciones en el patrón de erupción son dependientes de la edad, ya que los dientes recién mencionados aparecen dentro de un promedio de edades comprendido entre los seis y los ocho aîos. En cambio, en caninos y premolares son los dientes en los que se observaron mayores diferencias en los tiempos de erupción, siendo éstos los que suelen aparecer después de los once años, hasta los quince, esto
H.S.D. = Hemiarcada superior derecha.

H.S.I. = Нелiarcada supcrior izquierda.

H.I.D. = Hemiarcada inferior derecha.

H.I.L. = Hemiarcada inferior izquierda. es, en la adolescencia. Alternativamente, este hallazgo podria ser consecuencia de la gran varianza observada, es decir, el tiempo que requiere un individuo con $S$. de Down para que su erupción se complete sería mayor que la de un individuo normal, para cada pieza dentaria. Para someter a prueba ambas hipótesis, sería necesario aumentar el tamaño de la muestra de pacientes con S. de Down, ya que si el problema fuese simplemente muestral, debería disminuir la varianza.

Se puede concluir que una de las principales diferencias entre los individuos con S. de Down y los normales es la mayor varianza observada entre los niños con el trastomo ${ }^{6,12}$. Sin embargo, los niños Down tienen un retraso característico en su erupción dentaria permanente, la cual mantiene una similaridad con la secuencia des. crita para población normal. 
Tabla 4

Secuencia cronológica de erupción de la dentición pesmanente en población chilena nermal (*) (+)

\begin{tabular}{|c|c|c|c|c|c|c|c|c|c|c|c|}
\hline \multicolumn{6}{|c|}{ Niños } & \multicolumn{6}{|c|}{ Niñas } \\
\hline H.S.D. & $x$ & D.T. & H.S.I. & $x$ & D.T. & H.S.D. & $\mathrm{x}$ & D.T. & H.S.I. & $\mathrm{x}$ & D.T. \\
\hline Ml & 76.55 & 10,92 & M1 & 76,09 & 10,94 & M1 & 73,04 & 9,68 & M1 & 71,91 & 16,90 \\
\hline IC & $8 ? .72$ & 10,18 & IC & 87,36 & 11,06 & IC & 85.47 & 11,25 & IC & 85,49 & 11,54 \\
\hline IL & 99,59 & 15.02 & IL & 98,89 & 18,69 & IL & 96,32 & 9,60 & IL & 95,58 & 10,23 \\
\hline PM1 & 122,49 & 20.80 & PM1 & 125.64 & 18,94 & PMI & $11\}, 18$ & 16.25 & PM1 & 118,41 & 16,87 \\
\hline PM2 & 132,20 & 18.50 & PM2 & $135,1 ?$ & 20,59 & PM2 & 128,14 & 19,93 & C & 126.96 & 15,61 \\
\hline C. & 137,37 & 16,51 & $c$ & 137,25 & 18,52 & C & 129,48 & 18,70 & PM2 & 127,29 & 17,26 \\
\hline $\mathrm{M} 2$ & 148,47 & 14,16 & $\mathrm{M} 2$ & 148,74 & 14,39 & M2 & 144,13 & $18,9 ?$ & M2 & 143.00 & 16.46 \\
\hline H.I.D. & $\mathrm{X}$ & D.T. & H.I.I. & $\mathrm{x}$ & D.T. & H.L.D. & $\mathrm{x}$ & D.T. & H.I.I. & $\mathrm{X}$ & D.T. \\
\hline M1 & 69,56 & 23,69 & M1 & 70,48 & 20,10 & IC & 70,82 & 19,66 & M] & 70,78 & 8,31 \\
\hline IC & 72,58 & 11,9] & IC & 71,62 & 13,02 & $\mathbf{M} 1$ & 71,58 & 6,85 & IC & 72,44 & 17,90 \\
\hline lL & 89,36 & 12,91 & IL & 88,50 & 10,86 & IL & 84,95 & 13,54 & IL & 86,42 & 12,40 \\
\hline PM1 & 128,60 & 19,00 & PM1 & 126.40 & 17,26 & C & 117,94 & 12,56 & C & 119,24 & 14,44 \\
\hline C & 129,02 & 19.07 & $\mathrm{C}$ & 129,8 ? & 15,44 & PM1 & 119,62 & 19,34 & PM1 & 121,98 & 16,14 \\
\hline PM2 & 139,52 & 19.16 & PM2 & 135,8 ? & 17,46 & PM2 & 130,35 & 20,04 & PM2 & 129,21 & 23,08 \\
\hline M2 & 140,73 & 15.51 & $\mathrm{M} 2$ & 140,32 & 15.39 & $\mathrm{M} 2$ & 133,44 & 15,14 & M2 & 133,44 & 16,06 \\
\hline
\end{tabular}

- Datos de Cionzález, M.F., 1988.

$+\quad$ Ldad en meses.

$\mathrm{IC}=$ Incisivo central

$\mathrm{IL}=$ Incisivo lateral

C. $=$ Canino

PM $]=$ jer premolar

$\mathrm{PM} 2=2^{\circ}$ premolar

Ml = 1er molar

M2 $=2^{\circ} \mathrm{molar}$

\section{Resumen}

Se examinaron 240 pacientes con sindrome de Down, que asisten a escuelas especiales de Santiago de Chile, con el propósito de determinar la cronologia de erupción de la dentición definitiva y compararla con el patrón de erupción de la población chilena nomal. Los nif̃os con síndrome de Down presentan en el maxilar superior e inferior un retraso significativo de la erupción de algunos dientes; éstos son los siguientes: en el maxilar superior, el primer molar derecho $(85,35 \pm 20,03$ meses $)$ e izquierdo $(87,41 \pm 22,37$ meses $)$, primer y segundo premolar izquierdo $(161,60 \pm 60,43$ y $172,10 \pm 79,57$ meses, respectivamente) y canino izquierdo $(163,72 \pm 81,55$ meses). En el maxilar inferior, el primer molar derecho $(90,98 \pm 24,52$ meses $)$.
H.S.D. = Herniarcada superior derecha.

H.S.I. = Hemiarcada superior izquierda.

H.J.D. = Hemiarcada inferior derecha.

H.L.I. = Hemiarcada inferior izquierda. e] incisivo central derecho e izquierdo $(84,26 \pm$ 21,38 y 84,59 1 17,72 meses, respectivamente), el incisivo lateral derecho e izquierdo $(101,89 \pm$ 23,79 y $117,53 \pm 83,02$ meses, respectivamente) $y$ el canino izquierdo $(147,57 \pm 41,54$ meses). Las niffas con síndrome de Down presentan en el maxilar superior e inferior retraso significativo en la erupción de los siguientes dientes: en el maxilar superior, los segundos molares dere. cho e izquierdo $(172,71 \pm 83,02$ y $191,88 \pm$ 55,90 meses, respectivamente) y el primer molar ixquierdo $(84,48 \pm 19,65$ meses $)$. En el maxilar inferior, segundo molar y primer premolar derechos $(163,30 \pm 59,31$ y $131,07 \pm 21,94$ meses, respectivamente) y ambos incisivos laterales derecho e izquierdo $(102,27 \pm 52,86$ y $112,87 \pm$ 73,47 meses, respectivamente). La secuencia de la enupción dentaria superior e inferior en niños 


\section{NIÑOS}

MAXILAR SUPERIOR

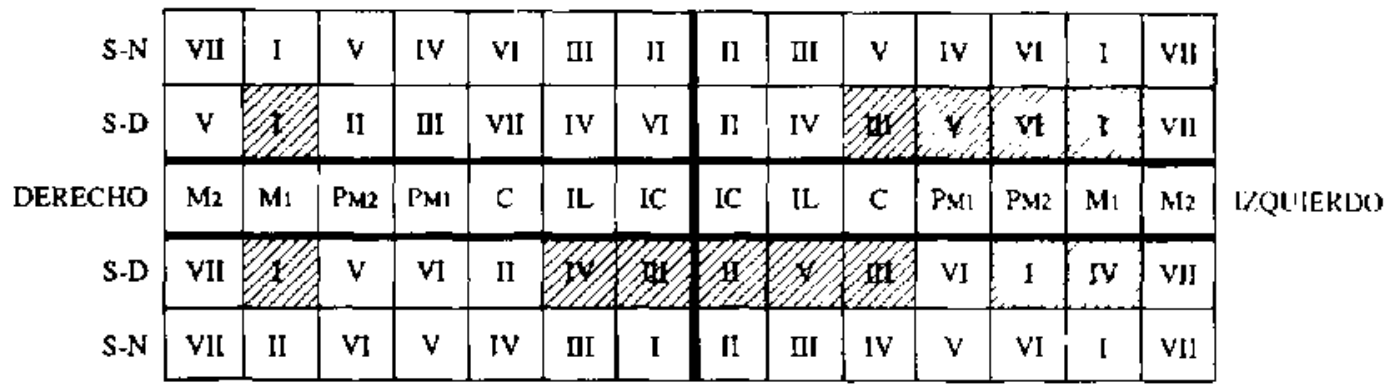

MAXILAR INFERIOR

$\mathbf{S}-\mathbf{N}=$ Secuencia normal.

S.D $=$ Secuencia Down

$Z A$ = Tiempo de erupción con retraso significativo.

* = Números romanos indican secuencia de exupción.

\section{NIÑAS}

MAXILAR SUPERIOR

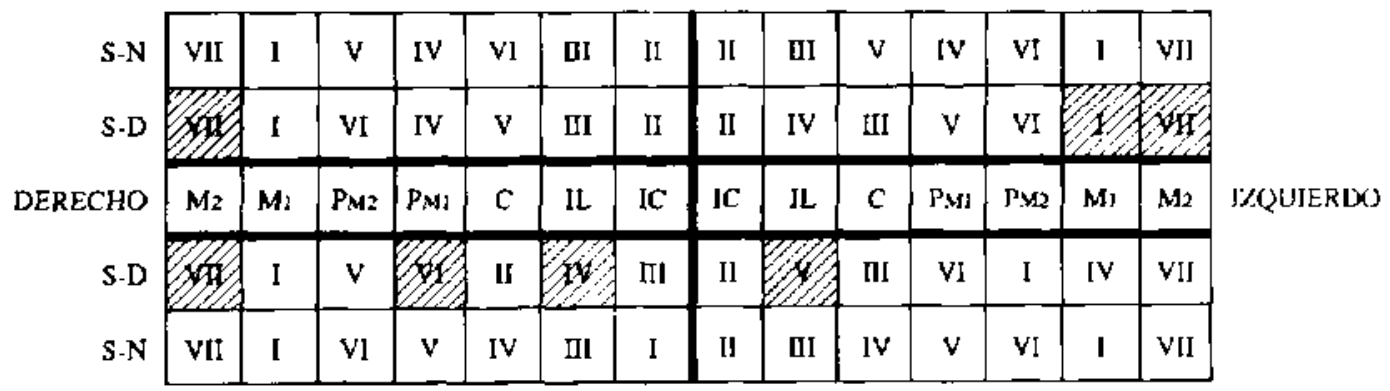

MAXILAR INFERIOR

Figara: Secuencia cronológica de la erupción dentaria permanente en individuos con sindrome de Down y normales.

$\begin{array}{llll}\text { IC } & \text { Incisivo central } & \text { PM2 } & 2^{\circ} \text { premolar } \\ \text { IL } & \text { Incisivo lateral } & \text { M1 } & 1 \text { er molar } \\ \text { C } & \text { Canino } & \text { M2 } & 2^{\circ} \text { molar }\end{array}$

PM1 ler premolar

con síndrome de Down comparada con la de la población normal presenta una gran asincronia que afecta a veinte dientes $(71,4 \%)$. En las niñas con sindrome de Down esta secuencia asincrónica afecta sólo a 15 dientes $(53,6 \%)$. Una de las principales diferencias entre los individuos Down y normales se debe a la mayor varianza observada en la muestra Down.
(Palabras clave: erupción dental, síndrome de Down.)

\section{Referencias}

1. Stewart RE and Poole AE: The orofacial st ructures and their association with congenital abnormalities. Pediatr Clin North Am 1982;29:547-584. 
2. Smirh $D W:$ Recognizable patterns of human malformations $2^{\text {nd }}$ ed. Philadelphia, WB Saunder Co., 1976.

3. Oster $f$ : Mongolism. A clinicogenealogical investigation comprising 526 mongols living on Seealand and neighboring islands in Denmark, Copenhagen, Danish Science Press Ltd., 1953.

4. Spizzer $R$ and Quilian $R L$ - Observations on congenital anomalies in teeth and skull in two groups of mental defectives. Brit J Radiol 1958;31:596.

5. Cohen $M M$ and Winer $R A$; Dental and facial characteristhics in Down's syndrome (mongolism). J Dent Res 1965;44: 197-208.

6. Barkla $D H$ : Ages of eruption of permanent teeth in mongols. J Ment Defic Res 1966; 10: 190-197.

7. Otero $E$, Sznadjer $N$ : Agenesia de dientes petmanentes en el Síndrome de Down (mongolismo). Rev Asoc Odont Argent 1966; 54: 257-259.

8. Roche $A F$ and Barkin $D H$. The development of the dentition in mongols. Aust Dent J 1967; 12: 12-16.

9. Spitzer $R$ : Observations on congenital dentofacial disorders in nongolism microcephaly. O.S., O. M. \& O.P. 1967;24:325-332.

10. Orner $G$ : Congenitally absent permanent teeth among mongols and their sibs. J Ment Defic Res 1971:15: 292-302.

11. Cohen MH, Sr, and Cohen MM Jr: The oral manifestations of trisomy $G$. (Down Syndrome). Birth Defects 1971;7:241.
12. Orner $G$ : Esuption of the permanent leeth in mongoloid children and their sibs. J Dent Res $1973 ; 52$ : 1202-1208.

13. Orner $G$ : Posteruptive tooth age in children with Down's Syndrome and their sibs. I Dent Res $1974 ; 54: 581-587$.

14. Molina I, Viñas L, Garcia-Godoy FM: Erupción clínica de los dientes permanentes en nimos con impedimentos mentales de Santo Domingo. Acta Odontol Pediatr 1982; 3:69-73.

15. Garcia $B C$, Mas $B C$, Pérez FD: Una aportación al estudio de las canes en escolares españoles afectados de Trisomía 21 . Avances en Odontoestoma. tología 1985; I : 137-146.

16. Jara $L$, Ondorza A, Infante JI et al.: Anomalías orofaciales en pacientes con Sindrome de Down en una muestra de población chilena, Rev Chil Pediatr 1986:57: 510-513.

17. González ME: Cronología y secuencia de erupción de la dentición permanente $y$ su relación con la presencia de dos marcadores genéticos: Tubércu. lo de Carabelli y Mesiogiro-versión, en escolares del Area Metropolitana Norte. Tesis Fac Odont Univer de Chile 1988.

18. Valenzuelo $C$, Canals $M$, Vergora $P$ : Maduración ósea de niưos de 0 a 6 años. Muñeca y Mano. Il Parte. Análisis de probitos para huesos aislados. Rev Chil Pedjatr 1985; 56 : 329-333.

Esta publicación está disponible en copias de microfilms de 16 y $35 \mathrm{~mm}$ y microfichas de $105 \mathrm{~mm}$, las que pueden solicitarse a:

University Microfilms International

300 North Zeeb Road

Ann Arbor, Michigan 48106, USA.

This journal is also available in $16 \mathrm{~mm}$ microfilm. $35 \mathrm{~mm}$ microfilm and $105 \mathrm{~mm}$ microfilm copies through

University Microfilms Internationat,

300 North Zeeb Road.

Ann Arbor, Michigan 48106, USA 